\title{
Face recognition using local geometrical features - PCA with Euclidean classifier
}

\begin{abstract}
The goal of this research is to get the minimum features and produce better recognition rates. Before doing the feature selection, we investigate automatic methods for detecting face anchor points with 412 3D-facial points of 60 individuals. There are 7 images per subject including views presenting light rotations and facial expressions. Each images have twelve anchor points which are Right Outer Eye, Right Inner Eye, Left Outer Eye, Left Inner Eye, Upper nose point, Nose Tip, Right Nose Base, Left Nose Base, Right Outer Face, Left Outer Face, Chin, and Upper Face. All the control points are based on the measurement on an absolute scale $(\mathrm{mm})$. After all the control points have been determined, we will extract a relevant set of features. These features are classified in $3:$ (1) distance of mass points, (2) angle measurements, and (3) angle measurements. There are fifty-three local geometrical features extracted from 3D points human faces to model the face for face recognition and the discriminating power calculation is to show the valuable feature among all the features. Experiment performed on the GavabDB dataset (412 faces) show that our algorithm achieved $86 \%$ of success when respectively the first rank matched.
\end{abstract}

